\title{
DEEP NECK INFECTIONS OF ODONTOGENIC ORIGIN AND THEIR CLINICAL SIGNIFICANCE. A RETROSPECTIVE STUDY FROM HRADEC KRÁLOVÉ, CZECH REPUBLIC
}

\author{
Helena Doležalován ${ }^{1}$ Josef Zemek ${ }^{2}$, Luboš Tuček ${ }^{1}$
}

Department of Dentistry, Faculty of Medicine, Charles University, and University Hospital in Hradec Králové, Czech Republic $^{1}$; Department of Computer Systems, University Hospital in Hradec Králové, Czech Republic ${ }^{2}$

\begin{abstract}
Summary: Introduction: Cellulitis remains a very serious disease even today. Mortality, which varied between 10-40\%, has been reduced owing to the standard securing of airway patency and use of an appropriate surgical treatment approach. Materials and methods: A total of 195 patients were hospitalised for cellulitis at the University Hospital in Hradec Králové during 2007-2011. The following parameters were evaluated: age, gender, dependence of incidence of the disease on the season of the year, frequency of attacks of the particular areas and their clinical characteristics, aetiology of the inflammation, types of patient complaints, prevalence of current systemic diseases, results of microbiological and selected laboratory analyses, socio-economic status of the patients, and duration of patient stay at the hospital. Statistical analysis was performed by using Pearson's correlation coefficient, the statistical significance level was $\mathrm{p}<0.05$.

Results: The mean age of the patients was 39.8 years. The group of 195 patients included 108 (55\%) males and 87 (45\%) females. The mean time between the first symptoms of the disease and admission to the Department was 5 days. From among the 195 patients, $116(59.5 \%)$ were working persons, 79 (40.5\%) were non-working (children, students, unemployed persons, women on maternity leave, retired people). The odontogenic origin of the disease was verified in $173(88.7 \%)$ patients. In total, $65(33.3 \%)$ patients had no coinciding complicating systemic disease, $22(11.3 \%)$ patients had diabetes mellitus. The most frequent symptom of cellulitis was painful swelling, found in 194 (99.5\%) patients, followed by jaw contracture, found in $153(78.5 \%)$ patients.

Conclusion: The results are largely very similar to those of previous studies performed in other countries, except that we found no correlation between the prevalence of cellulitis and the socio-economic status, nor have we confirmed Klebsiella pneumoniae sp. as the cause of cellulitis in patients with diabetes mellitus.
\end{abstract}

Keywords: Cellulitis; Odontogenic infection; Oral microflora; Bacteria

\section{Introduction}

Even today, inflammatory processes in maxillo-facial areas - cellulitis, or deep neck inflammations, are regarded as very serious diseases that can threaten patient's health and life. Such inflammations can be categorised aetiologically into inflammations of odontogenic origin and inflammations of non-odontogenic origin. The former predominate (70-90\%) in adult patients, whereas the latter predominate in paediatric patients.

Cellulitis is largely caused by mixed aerobic/anaerobic bacterial microflora, which routinely colonises the oral cavity and does not primarily consist of pathogenic microbial species. Clinically, cellulitis includes mostly suppurative inflammation, with the tendency to delimiting and formation of abscesses. However, cellulitis can also develop into a formation of phlegmonous nature and can then propagate rather readily into anatomically quite remote preformed areas in the head and neck area. Older published data postulated that the incidence, severity, morbidity and mortality of cellulitis have decreased significantly during the past decades (1). This information is in direct contradiction to our current clinical experience. The amount of cellulitis is increasing with more severe clinical course. Among the factors that contributed to this success were early diagnosis, introduction of radical surgical treatment and use of modern, highly efficacious antibiotics. Despite this major advance in the treatment of cellulitis, we must be prepared at any moment to face an unexpected situation and/or complications that can accompany cellulitis therapy (including enhanced bacterial resistance and the associated insensitivity of a number of bacterial agents to various antibiotics). Once appeared, cellulitis complications are a constant cause of very high $(50 \%)$ mortality (2).

When assessing the severity and prognosis of cellulitis, attention must be paid, in particular, to warning signs indicating propagation of infection through the body, such as body temperature increase above $39^{\circ} \mathrm{C}$, appreciable 
jaw contracture, signs of inflammation spreading to the surroundings along the large neck vessels, oedema or skin reddening in the jugular area, pain under the sternum, but also swollen eyelids and retrobulbar pain, stridor, cough and pain in the chest $(3-5,1)$. Appropriate timing of surgical intervention is an important factor in cellulitis therapy: it should be conducted early and correctly, adhering (also today) to the rule "Ubi pus, ibi evacuo..."

No recent information on the situation in the incidence, severity, cause(s) and occurrence of complications in cellulitis of odontogenic origin in the Czech Republic is available except for one paper focusing on the issue of severe complications accompanying deep neck inflammations (6). Therefore the authors of the present paper performed analysis of this severe disease in a group of patients treated during the 5-year period of 2007-2011 at the authors' workplace, i.e. the Maxillo-facial Surgery Unit, Department of Dentistry, Faculty of Medicine, Charles University, and Teaching Hospital in Hradec Králové, Czech Republic, where the majority of patients with this disease from the East Bohemian region have been treated. The authors of this contribution also compared their own findings with those recently published by other authors.

\section{Material and methods}

The study was approved by local ethical commitee. A total of 195 inpatients with the diagnosis of cellulitis were treated at the Maxillo-facial Surgery Unit, Department of Dentistry, Faculty of Medicine, Charles University, and Teaching Hospital in Hradec Králové, during 2007-2011. Outpatients with similar diagnoses were not included in the study. All the patients were examined by the adequate routine procedure, and where their health permitted so, subjected to panoramic X-raying and ultrasound examination. The results were entered into the patient's medical records and the patient was then referred to the maxillo-facial surgery unit for admission. The patient's medical history record was then compiled, including the diagnosis with which the patient had been referred, the name of the referring physician, length of the disease from the first symptoms to the date of admission, any previous medical treatment and its effect, past tooth extractions, final diagnosis of some form of cellulitis and the treatment plan. Prior to the surgery, performed in general anaesthesia, the patients were subjected to routine preoperative assessment, including examination at the anaesthesiology centre. The centre decided if and how the patient can be intubated. Many patients were intubated by the flexible fibroscopic method. Therefore they were transferred to the inpatient ward of the Department of Anaesthesiology, Resuscitation, and Intensive Medicine (KARIM), where they were extubated later, usually during the next 48 hours. In some clear cases where abscess centres were present without previous radiographic examination. We performed external incision and drainage under local anaesthesia. All patients in whom intraoral mucous membrane incision was feasible were treated in local anaesthesia. Pearson's correlation coefficient was used for statistical evaluation of the parameters. Statistical analysis between elevated body temperature and the CRP level, body temperature and the leukocyte count, leukocyte count and the CRP level, duration of the cellulitis treatment and the leukocyte, duration of the treatment on the CRP level. The statistical significance level was $\mathrm{p}<0.05$.

\section{Results}

The group of patients included in this study comprised $108(55 \%)$ males and 87 (45\%) females (Graph 1). The number of cellulitis inpatients was highest in February (25 patients) and in June (24 patients) and lowest in November (5 patients) (Graph 1). The number of inpatients treated during a defined time period was steady during the years included, only in 2009 it was appreciably lower. The mean patient age and the median were 39.8 years and 36 years, respectively. The youngest patient was aged 4 , the oldest patient 91. The group included 116 employed and 17 un-

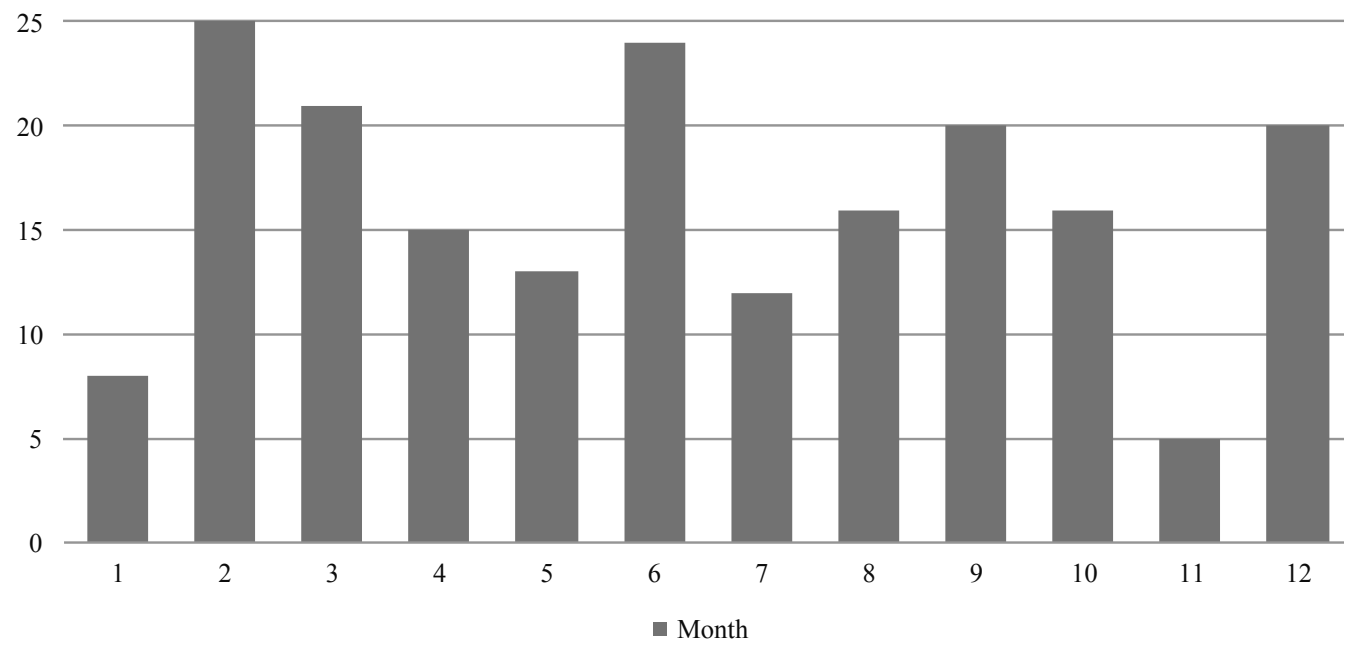

Graph 1:

Distribution of the patients throughout the year seasons. 


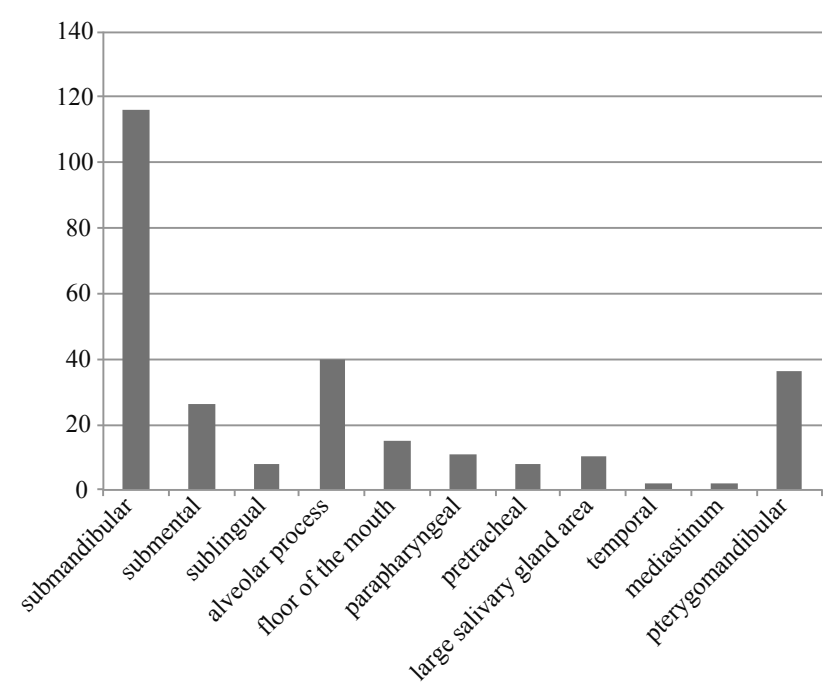

Graph 2: Affected areas.

employed adults plus 11 children, 10 students, 24 retired persons, 10 disability pension recipients, and 7 women on maternity/parent leave. The mean length of stay was 8 days, median 7 days. The shortest length of stay was 2 days: this concerned 3 patients who, after their health had improved, demanded immediate discharge from the hospital to home care at their own risk and were discharged after signing the treatment refusal form. The longest stay was 62 days: this concerned a polymorbid patient in whom cellulitis was complicated by mediastinitis. The mean time from the first symptoms to patient admission to the ward was 5 days, median 4 days (during that time the patients usually expected their health to improve spontaneously or they were treated by other health centres and by inadequate methods). In some cases the patients came to this Department only three weeks after the first symptoms of the disease.

The maxillo-facial abscess largely affected the submandibular area (Graph 2). More than one maxillo-facial space was affected in a number of patients. Odontogenic aetiology, which was the most frequent cause of the cellulitis, was identified in $173(88.7 \%)$ patients. In 9 cases the cellulitis was caused by complicated purulent sialoadenitis and in 3 cases, by complications of purulent lymphadenitis. In isolated cases, the cellulitis was due to unrecognised mandibular fracture, tumour or furuncle. From our patients' health assessment we concluded that only 65 (33.3\%) patients were free of any complicating systemic disease. A total of $40(20.5 \%)$ patients had been treated for cardiovascular diseases and $22(11.3 \%)$ patients were diabetics. Among those 22 diabetic patients, diabetes mellitus had not been recognised in 3, and associated congenital pancreatic atrophy was diagnosed in a 16 year old diabetic girl. Drug abuse, including intravenous narcotics, was admitted by 5 patients. None of the patients was HIV positive. Where patients with decompensated systemic diseases were involved, appropriate specialists, mostly diabetologists, internal medicine specialists or rheumatologists, were consulted regarding the therapy.

The majority of patients 194 (99.5\%) complained of pain and oedema in the affected anatomic area at the time of admittance. Jaw contracture of various degrees was the next frequent $(153,78.5 \%)$ sign of cellulitis, followed by swallowing problems - odynophagia $(125,63.9 \%)$ and increased body temperature $(117,60.0 \%)$. Respiratory problems of various degrees were recorded in 10 patients. Isolated cases of neck movement disorders $(2 \times)$ and dysphonia $(1 \times)$ were observed (Graph 3).

In addition to the basic biochemical parameters, C-reactive protein levels, glycaemia, and blood counts were measured in all patients on the day of admission. Statistical analysis provides medium strong correlations between elevated body temperature and the CRP level $(r=0.27)$ and between body temperature and the leukocyte count $(\mathrm{r}=0.26)$. A high correlation, however, was identified between the leukocyte count and the CRP level $(r=0.43)$. No correlation between the duration of the cellulitis treatment and the leukocyte count was observed $(\mathrm{r}=0.21)$. A medium

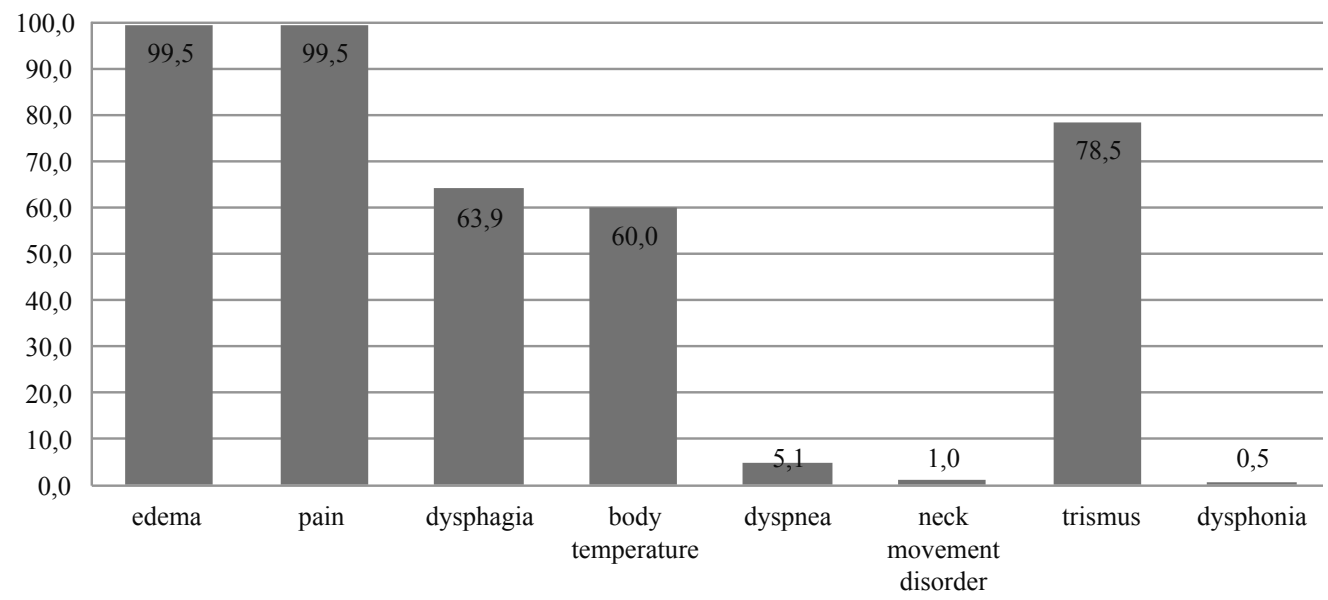

Graph 3:

The most frequent signs of cellulitis $(\%)$. 
dependence of the duration of the treatment on the CRP level was found $(\mathrm{r}=0.34)$.

Ultrasound examination and computed tomography were indicated in $20.5 \%$ and $12.8 \%$ of patients, respectively, within the examination on admission. In the remaining patients, cellulitis was diagnosed and therapy indicated based on clinical examination and a panoramic photograph of the jaws. Our group included $23(11.8 \%)$ patients who reported an allergy to penicillin.

Antibiotics used within the cellulitis therapy were selected based on clinical condition severity and disease development. All antibiotics were administered intravenously. Where the results of bacteriological culture analysis were not available, the antibiotics were selected empirically. Ampicillin potentiated with clavulanic acid or cefuroxim was used in monotherapy. Where two different antibiotics were used in combination, one of the above antibiotics was usually combined with metronidazole. Three antibiotics in combination, viz. two from those mentioned above plus gentamicin, were used in the most severe cases. In patients with premonitory signs of inflammation. After obtaining the results of bacteriological analysis, the efficacy of the empirically selected antibiotics was assessed based on the antibiogram. Medication had to be changed only in those patients who stayed at the intensive care unit longer than 5 days after surgery (see Table 1 for details).

A total of 74 (38.0\%) patients were treated conservatively (i.e. incision was unnecessary).

The necessity of intensive conservative treatment with parenteral antibiotics led to increased number of inpatient cases in our cohort of patients. Also the possibility of early surgical intervention when needed could be guaranteed when the patient is in the hospital.

Surgery was performed in $121(62.1 \%)$ patients. This included intraoral incision (43 patients, who constituted $35.5 \%$ of the surgically treated patients and $22.1 \%$ of all patients included in the group) and revision and drainage of the maxillo-facial space from external access (78 patients, who constituted $64.5 \%$ of the surgically treated patients and $40.0 \%$ of all patients included in the group) - see Graph 4 . Extractions of the teeth which caused the disease are not included among the surgeries. From among the surgically treated patients, $93(76.9 \%)$ were operated on the day of admission, the remaining patients, during the first to fourth day of stay at the hospital. Two patients who were treated for severe necrotizing fasciitis were subjected to a series of surgeries. Fourteen patients were transferred to the intensive care unit after the surgery. A total of 9 patients were ventilated for $24-48$ hours. In another 4 patients in very severe condition, the time of artificial lung ventilation was longer than 48 hours, temporary tracheotomy was performed after the surgery, and the patients were decanulized only before dismission (data from those patients are summarised in Table 1). For patients in whom cellulitis was complicated by mediastinitis, the surgery was performed in cooperation with a thoracic surgeon. The surgical revision of all adjacent spaces was indicated in case of phlegmone. Upper mediastinotomy was performed upon mediastinal spreading. Thoracic surgeon took part in involvement of deeper mediastinal structures. Complications in the patient group included

Tab. 1: ICU patients characteristics.

\begin{tabular}{|c|c|c|c|c|}
\hline Gender & Female & Male & Male & Male \\
\hline Age & 57 & 62 & 35 & 43 \\
\hline General health & Corticosteroids & Diabetes & Coxarthrosis & $\begin{array}{l}\text { Diabetes } \\
\text { Pancreatitis } \\
\text { Hepatopathy }\end{array}$ \\
\hline Aetiology & Odontogenic & Odontogenic & Odontogenic & Odontogenic \\
\hline Body temperature & 39.4 & 36.6 & 39.1 & 35.6 \\
\hline Leukocytes & 20.01 & 22.3 & 16.88 & 7.84 \\
\hline CRP & 254 & 370 & 278 & 204 \\
\hline APV & 13 & 19 & 4 & 8 \\
\hline ICU & 17 & 25 & 12 & 15 \\
\hline Total duration of stay & 31 & 62 & 34 & 25 \\
\hline Complications & $\begin{array}{l}\text { Sepsis } \\
\text { MODS }\end{array}$ & $\begin{array}{l}\text { Mediastinitis } \\
\text { Pneumonia }\end{array}$ & $\begin{array}{l}\text { Pleural effusion } \\
\text { Effusion }\end{array}$ & $\begin{array}{l}\text { Pleural effusion } \\
\text { Mediastinitis } \\
\text { Pneumonia }\end{array}$ \\
\hline Microbiology & Streptococcus spp. & $\begin{array}{l}\text { Staphylococcus spp. } \\
\text { Enterococcus spp. } \\
\text { Peptosterptococcus spp. } \\
\text { Peptostreptococcus spp. } \\
\text { Bacteroides spp. }\end{array}$ & $\begin{array}{l}\text { Staphylococcus spp. } \\
\text { Peptostreptococcus spp. } \\
\text { Bacteroides spp. } \\
\text { Propionibacterium spp. } \\
\text { Enterobacter spp. }\end{array}$ & $\begin{array}{l}\text { Burkholderia spp. } \\
\text { Stenotrophomonas spp. } \\
\text { Candida albicans } \\
\text { Candida tropicalis }\end{array}$ \\
\hline
\end{tabular}


mediastinitis, pneumonia and pleural effusion in 2 patients $(1.0 \%)$. Signs of sepsis were diagnosed in $4(2.1 \%)$ patients. The multiple organ dysfunction syndrome developed in one $(0.5 \%)$ patient who had been on long-term corticosteroid therapy for another disease. None of the patients in the study group died. Permanent consequences of the cellulitis treatment only included scarring on the skin and the loss of teeth that were the root of the disease.

The results of microbiological examination were available retrospectively for 74 patients. The culture was negative in $12(16.2 \%)$ patients and positive in $62(83.8 \%)$ patients. Various species of the bacterial genera Streptococcus, Staphylococcus, Peptostreptococcus, Propionibacterium, Haemophilus, Stenotrophomonas, Burkholderia, Enterobacter, Klebsiella, Pseudomonas, Escherichia, Lactobacillus, Prevotella, Neisseria, Actinomyces, Veillonella, Fusobacterium, Enterococcus were identified. Yeasts of the genus

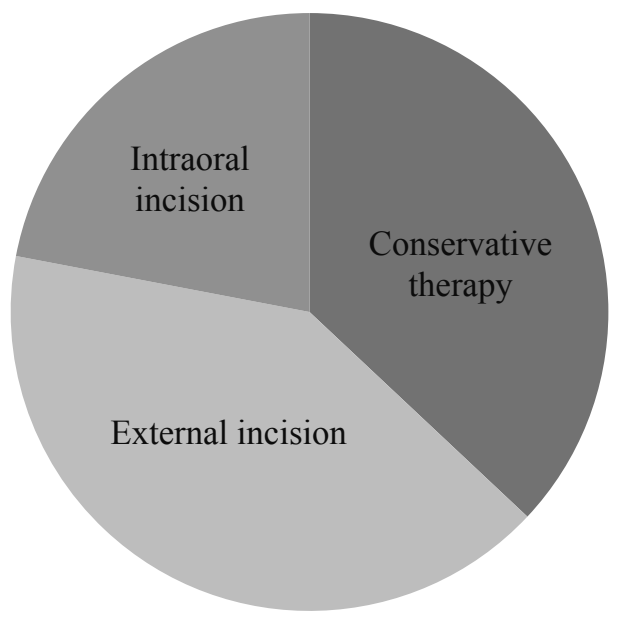

Graph 4: Cellulitis therapy modalities.
Candida were also identified in 3 cases (C. albicans $2 \times$, C. tropicalis $1 \times)$. Somewhat atypical microbiological results were obtained for 4 patients (Table 1). A triazole antimycotic (fluconazole) was administered systemically to patients in whom mycotic infection had been identified. Patients with the cervico-facial form of actinomycosis were treated with benzyl-penicillin. An overview of the microbiological findings is presented in Graph 5.

\section{Discussion}

The results of this study of cellulitis patients were compared to those of similar studies from other hospitals, where streptococci, staphylococci and prevotellae were identified as the major pathogenic microorganisms (2, 3, 5, 7-15). This is borne out by our observations. However, while some papers $(3,5,12,14)$ mention Klebsiella pneumoniae as a significant infectious agent for cellulitis in diabetics, we observed this microorganism only twice in our patient group, and those 2 individuals were not diabetics. The frequency at which the various maxillo-facial spaces were affected was also in line with published data $(1,3,6,10,11,16,17)$. Neither the therapeutic procedures nor the types of the empirically selected antibiotics were different either.

The frequency of severe complications, especially mediastinitis and septic conditions, was similar to that observed by Czech otorhinolaryngologists (6), who examined a group of 634 patients, among whom mediastinitis was present in $6.6 \%$ and mortality was $0.6 \%$.

According to some authors (7), cellulitis more frequently affects individuals from socially and economically disadvantaged populations. This conclusion was not confirmed by our study. In our group, there were only 17 unemployed as already mentioned. We explain this, among other factors, by the generally insufficient information and motivation and

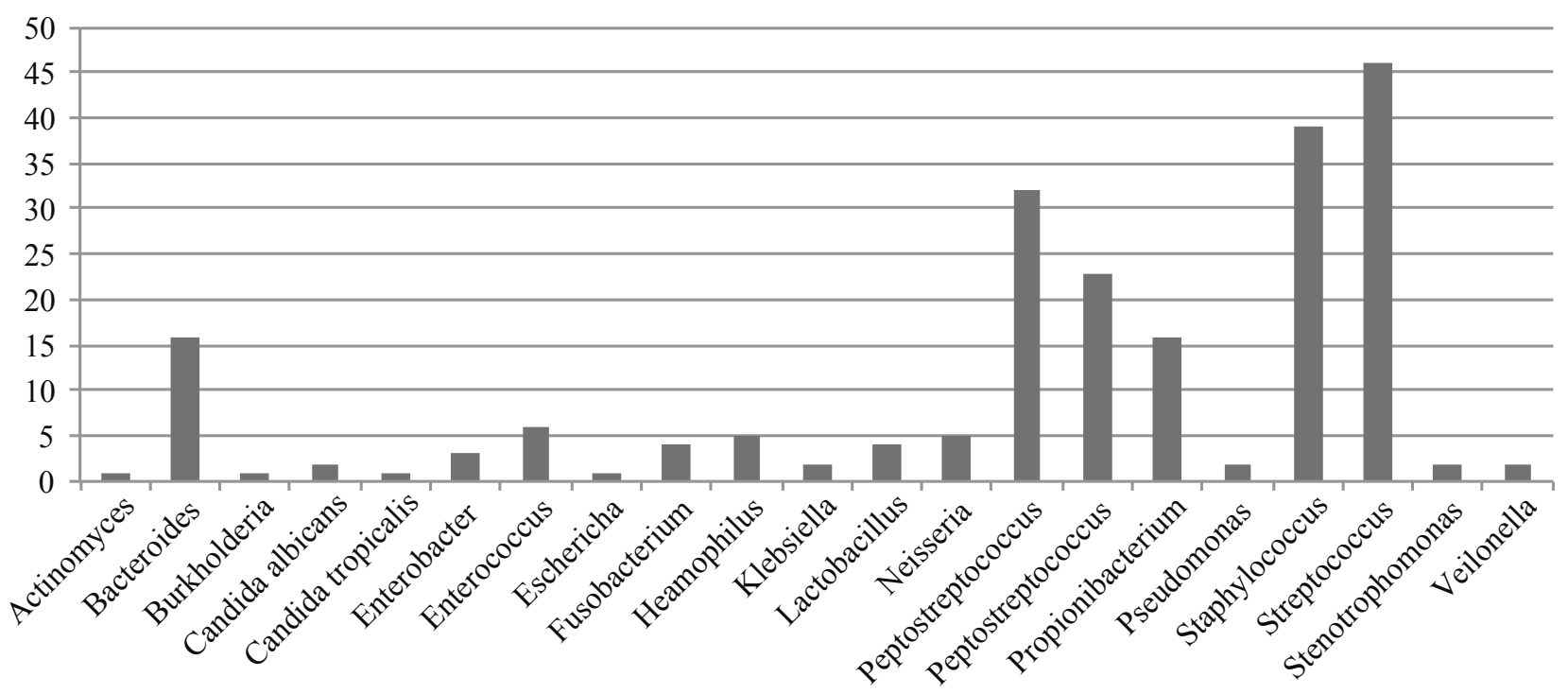

Graph 5: Microorganisms in the cultures. 
the associated poor level of oral hygiene and hence, poor oral health, still existing in a large fraction of the Czech population.

Modern imaging methods such as magnetic resonance and positron emission tomography are quite frequently included among examination algorithms for patients with cellulitis in developed countries $(3,5,6,12,18)$. Although those techniques are relatively well available and their use in cellulitis diagnosis is feasible in the Czech health sector as well, routine diagnosis of cellulitis at our department remains based on proven classical imaging methods, which are thus far more readily available and less expensive.

We frequently encountered situations where the dental practitioners hesitated and failed to resolve to extract the target tooth, even though the diagnosis of cellulitis was quite clear at that time. It is clear that this "let's wait" approach, consisting in administration of oral antibiotics and trepanation of the tooth, cannot be successful in cases of developing or fully developed cellulitis (in fact, postponing the tooth extraction procedure till the patient's conditions improves appreciably is warranted only in exceptional cases where the tooth is actually inaccessible). Only timely elimination of the cause associated with incision and drainage of the focus of the abscess (if present) will prevent further propagation of infection to the surrounding anatomic areas and reduce the time of treatment appreciably. This requires perfect knowledge of the topographic-anatomic situation in the maxillo-facial spaces and areas where the inflammation exudate is typically present. This is based on the clinical finding and ultrasound examination. Computed tomography may be of assistance in this as well.

\section{Conclusion}

In conclusion, cellulitis continues to be a frequent and rather severe disease of odontogenic origin in the maxillo-facial area. Its incidence can apparently be reduced only by fundamental "oral health" improvement among the Czech population as a whole, and also by adequate, i.e. timely and good quality treatment of teeth with "banal" diseases, particularly caries and its complications, which unless adequately treated as outlined above, result in infection transfer to the surrounding tissues and sometimes in the induction of serious diseases endangering patient health and life.

\section{Acknowledgements}

This study was conducted within the PRVOUK P37/13 Project.

\section{References}

1. Peterson LJ. Contemporary management of deep infections of the neck. J Oral Maxillofac Surg 1993 April; 51(3): 226-31.

2. Poeschl PW, Spusta L, Russmueller G, et al. Antibiotic susceptibility and resistance of the odontogenic microbiological spectrum and its clinical impact on severe deep space head and neck infections. Oral Surg Oral Med Oral Pathol Oral Radiol Endod 2010 August; 110(2): 151-6.

3. Boscolo-Rizzo P, Da Mosto MC. Submandibular space infection: a potentially lethal infection. Int J Infect Dis IJID 2009 May; 13(3): 327-33.

4. Santos Gorjón P, Blanco Pérez P, Morales Martín AC, et al. Deep neck infection. Review of 286 cases. Acta Otorrinolaringológica Esp 2012 February; 63(1): 31-41.

5. Lee J-K, Kim H-D, Lim S-C. Predisposing factors of complicated deep neck infection: an analysis of 158 cases. Yonsei Med J 2007 February 28; 48(1): 55-62. 6. Celakovsky P, Kalfert D, Tucek L, et al. Deep neck infections: risk factors for mediastinal extension. Eur Arch Oto-Rhino-Laryngol 2014 June; 271(6): 1679-83.

7. Agarwal AK, Sethi A, Sethi D, Mrig S, Chopra S. Role of socioeconomic factors in deep neck abscess: A prospective study of 120 patients. Br J Oral Maxillofac Surg 2007 October; 45(7): 553-5.

8. Al-Qamachi LH, Aga H, McMahon J, Leanord A, Hammersley N. Microbiology of odontogenic infections in deep neck spaces: a retrospective study. Br J Oral Maxillofac Surg 2010 January; 48(1): 37-9.

9. Boyanova L, Kolarov R, Gergova G, et al. Anaerobic bacteria in 118 patients with deep-space head and neck infections from the University Hospital of Maxillofacial Surgery, Sofia, Bulgaria. J Med Microbiol 2006 September; 55(Pt 9): 1285-9.

10. Har-El G, Aroesty JH, Shaha A, Lucente FE. Changing trends in deep neck abscess. A retrospective study of 110 patients. Oral Surg Oral Med Oral Pathol 1994 May; 77(5): 446-50.

11. Hasegawa J, Hidaka $H$, Tateda M, et al. An analysis of clinical risk factors of deep neck infection. Auris Nasus Larynx 2011 February; 38(1): 101-7.

12. Huang T-T, Liu T-C, Chen P-R, Tseng F-Y, Yeh T-H, Chen Y-S. Deep neck infection: analysis of 185 cases. Head Neck 2004 October; 26(10): 854-60.

13. Krautsevich L, Khorow O. Clinical aspects, diagnosis and treatment of the phlegmons of maxillofacial area and deep neck infections. Otolaryngol Pol Pol Otolaryngol 2008; 62(5): 545-8.

14. Rao DD, Desai A, Kulkarni RD, Gopalkrishnan K, Rao CB. Comparison of maxillofacial space infection in diabetic and nondiabetic patients. Oral Surg Oral Med Oral Pathol Oral Radiol Endod 2010 October; 110(4): e7-12.

15. Sakaguchi M, Sato S, Ishiyama T, Katsuno S, Taguchi K. Characterization and management of deep neck infections. Int J Oral Maxillofac Surg 1997 April; 26(2): 131-4.

16. Bakir S, Tanriverdi MH, Gün R, et al. Deep neck space infections: a retrospective review of 173 cases. Am J Otolaryngol 2012 February; 33(1): 56-63.

17. Smith JL, Hsu JM, Chang J. Predicting deep neck space abscess using computed tomography. Am J Otolaryngol 2006 August; 27(4): 244-7.

18. Umeda M, Minamikawa T, Komatsubara H, Shibuya Y, Yokoo S, Komori T. Necrotizing fasciitis caused by dental infection: a retrospective analysis of 9 cases and a review of the literature. Oral Surg Oral Med Oral Pathol Oral Radiol Endod 2003 March; 95(3): 283-90.

Received: 16/06/2015

Accepted in revised form: 09/09/2015

\section{Corresponding author:}

Helena Doležalová, MD, Department of Dentistry, Teaching Hospital Hradec Králové, Sokolská 581, 50005 Hradec Králové, Czech Republic; e-mail: helena.dolezalova@fnhk.cz 